\title{
Functional differentiation within hypothalamic behavioral systems in the cat
}

\author{
GARY G. BERNTSON and MICHAEL S. BEATTIE \\ Laboratory of Comparative and Physiological Psychology, The Ohio State University \\ 1314 Kinnear Road, Columbus, Ohio 43212
}

\begin{abstract}
A stimulation analysis was completed, by the use of roving stimulation electrodes, of an area in the cat hypothalamus where biting attack and threat behavior can be elicited. Results indicated that, while attack and threat have partially overlapping anatomical representations, there is considerable anatomical dissociation between these behaviors, even within overlap areas. Further, we found that a composite stimulation map, based on anatomical data pooled across animals, substantially overestimated the size of response areas and the degree of anatomical overlap between responses for a given animal. The results support the view that the hypothalamus contains partly overlapping, but functionally specific, mechanisms for different behaviors.
\end{abstract}

Since Hess's demonstration (see Hess, 1957) that electrical stimulation of the hypothalamus in cats can induce complex serially ordered behaviors, stimulation techniques have been employed widely in studying the neural substrates and functional organization of brain-behavioral systems (for reviews see Doty, 1969; Mogenson \& Huang, 1973; Roberts, 1970). Much of this work has indicated a high degree of anatomical differentiation and functional specificity within these neurobehavioral mechanisms (Roberts, 1969). For example, hypothalamic systems for different behaviors, in many cases, are regionally segregated (Roberts, 1970), and have anatomically differentiated projections (Bergquist, 1970; Berntson, 1972; Chi \& Flynn, 1971). Further, different hypothalamic response mechanisms can be selectively activated by appropriate thermal, hormonal, osmotic, or neurochemical stimulation (Andersson, 1953; Fisher, 1966; Grossman, 1969; Leibowitz, 1971; Roberts, Bergquist, \& Robinson, 1969). In addition, stimulation of hypothalamic behavioral mechanisms produces a highly lateralized facilitation of specific reflexes, which are functionally associated with the elicited behavior, suggesting the presence of a highly specific functional organization within these mechanisms (Flynn, Edwards, \& Bandler, 1971).

In contrast to this demonstrated specificity, many stimulation mapping studies of the hypothalamus and other brainstem areas (Bernston \& Hughes, 1974; Hess, 1957; Roberts, Steinberg, \& Means, 1967; Waldbillig, 1975; Woodworth, 1971), have shown that many behaviors can be elicited from fairly widespread areas, that there is considerable overlap in the anatomical distributions for some behaviors, and that, in some cases,

This research was supported by United States Public Health Service Grant MH-25630 to G. G. Berntson. M. S. Beattie was supported by National Institute of Mental Health Training Grant MH -06748 awarded to Donald R. Meyer. a single electrode can elicit more than one response. These latter findings have led some authors to question the anatomical and behavioral specificity of hypothalamic mechanisms and to suggest that the hypothalamus may serve as a relatively homogeneous motivational substrate capable of supporting a wide variety of behaviors (Valenstein, Cox, \& Kakolewski, 1970). Roberts and others (Roberts, 1969; Wise, 1968), however, have suggested an alternate explanation of these results based on overlapping distributions of functionally specific, but anatomically interdigitating systems for different behaviors.

It is likely, we believe, that much of the apparent nonspecificity in the distributions of behavioral substrates, and the overlap between behaviors, may be due to the common practice in mapping studies of "pooling" anatomical data from different animals. This "pooling" procedure, necessitated by the limited number of fixed electrodes which can be implanted into a single animal, can provide important information on the relative locations of behavioral systems. This procedure, however, tends to overestimate the anatomical extent of a behavioral substrate in a given animal and the degree of overlap between behavioral mechanisms, by including interanimal variability in the location of the mechanisms into the determination of the size of the effective substrates (Roberts, 1969). Individual anatomical variability of this sort has previously been demonstrated in the motor cortex (Asanuma \& Sakata, 1967; Penfield \& Jasper, 1954; Woolsey, Settlage, Meyer, Spencer, Pinto \& Travis, 1951), where a stimulation map of the representation of a selective muscle group, when based on a number of animals, can substantially overestimate the size of the relevant area in a single animal. Further, the procedure of pooling anatomical data disallows a fine-grained stimulation analysis of regions of overlap between behavioral systems in a single animal, which might reveal 
some degree of differentiation between behaviors in areas of overlapping representation.

In the present study, we employed "roving" stimulation electrodes, movable in the dorso-ventral dimension, to allow a fine-grained stimulation analysis of a region in the cat hypothalamus where threat behavior and predatory-like biting attack appear to have partially overlapping representations (Roberts \& Kiess, 1964; Wasman \& Flynn, 1962). We found a substantial degree of differentiation between these two behaviors, even within areas of anatomical overlap. We also found that, due to interanimal variability in the location of these behavioral mechanisms, a composite stimulation map based on pooled data substantially overestimated both the size of, and degree of overlap between, response mechanisms. These findings support the suggestion of Roberts (1969) and Wise (1968) that the hypothalamus contains anatomically and functionally specific systems for different behaviors.

\section{METHOD}

Eight cats were stereotaxically implanted, under sodium pentobarbital anesthesia, with unilateral (six cats) or bilateral (two cats) roving electrode assemblies aimed for the dorsal hypothalamus. The electrode assembly consisted of a $20-\mathrm{mm}$ length of 21-ga guide cannula attached to a drilled and tapped plastic hub, which could receive an electrode soldered to the end of a screw. The electrode was a No. 0 stainless steel insect pin, insulated with Epoxylite except for $.4 \mathrm{~mm}$ at the tip. When the electrode was inserted into the guide cannula and the screw engaged into the threaded hub, the electrode could be raised or lowered by advancing or withdrawing the screw. A clip attached to the screw served to connect the electrode to the stimulator. An indifferent electrode of stainless steel suture wire was embedded beneath the temporal muscle and attached to a socket. The electrode assembly and the indifferent electrode socket were secured to the skull with stainless steel screws and acrylic cement.

Following at least 1 week of postoperative recovery, all cats were given a stimulation test under free-moving conditions. Stimulation consisted of $50-\mathrm{Hz}$ 100-microsec monopolar cathodal pulses (Grass stimulator Model S4E). Voltage ind current levels were monitored on a calibrated oscilloscope (Tektronix 502A). Testing was carried out in a $73 \times 54 \times 48 \mathrm{~cm}$ Plexiglas box, containing a freshly killed rat. During the testing session, the electrode was progressively lowered in .5- or .6-mm steps, starting from at least $1 \mathrm{~mm}$ below the guide cannula. Each electrode locus was tested, at a wide range of voltage levels, for the elicitation of predatory-like biting attack on a rat, or threat behavior (hissing and/or growling, ear flattening, and sometimes clawing attack on a rat). Voltage levels were repeated as necessary to verify effects, and extended for each cat from below threshold for any overt response to a level where motor responses become so strong as to prevent further testing. Stimulation levels were increased as high as possible to insure that even high-threshold responses would not be overlooked.

After general testing, electrodes in six cats were repositioned to a positive locus, cemented in place, and retested after $24-48 \mathrm{~h}$ to determine the stability of behaviors over time.

Following completion of testing, animals were perfused with $10 \%$ formalin, their brains were removed, and frozen sections were cut at 50 microns. Alternate sections were stained with cresyl violet and luxol blue. Electrode tracks were then localized by direct projections of the sections onto atlas diagrams of Snider and Niemer (1961). The ventralmost extent of the electrode track was taken as the lowest stimulation locus. In most cases, the electrode at its ventralmost excursion exited the ventral surface of the hypothalamus, indicated by a precipitous drop in electrode impedance $(23 \%-67 \%)$ and subsequent confirmation by histological analysis. In these cases, the ventralmost hypothalamic stimulation point was localized at $.25 \mathrm{~mm}$ or $.3 \mathrm{~mm}$ above the ventral surface (one-half the advancement interval). To correct for possible shrinkage during histology, intermediate points were localized, along the electrode track, by interpolation between the lowest point and the indentation produced by the guide cannula. To facilitate comparison, all points are plotted on the median frontal plane for all electrodes (plane A10.0 of the atlas of Snider and Niemer. 1961).

\section{RESULTS AND DISCUSSION}

The anatomical and behavioral results of the present study are summarized in Figure 1, which shows the locations of electrode tracks in each animal and the behaviors elicited by each stimulation point along the track. The elicitation of different behaviors at different stimulation loci in a given animal appeared to result from the activation of stable behavioral systems rather than from dispositional or other changes taking place over time, since: (1) elicited behaviors were highly stable and reproducible within the period of testing at a given point, but would change immediately and dramatically when the electrode was lowered from one response locus to another; and (2) in the six cats tested, stimulation at positive loci $24-48 \mathrm{~h}$ after initial testing always yielded the same response or combination of responses as obtained initially.

\section{Overall Analysis}

Consistent with previous reports (Roberts \& Kiess, 1964; Wasman \& Flynn, 1962), there was often considerable anatomical overlap, both within and between animals, in the distributions of points yielding attack and those yielding threat (Figure 1). In spite of this overlap, there was considerable dissociation between these behaviors at many points. Of the 81 stimulation points producing biting attack or threat, $38(47 \%)$ produced only threat, $20(25 \%)$ yielded only attack, and only $23(28 \%)$ produced both. This double dissociation between attack and threat did not result simply from "pure" response points not being tested at sufficiently high intensities to reveal the second behavior, since the maximum stimulation intensities tested at "pure" and "mixed" points were not appreciably different (Table 1). Nor were pure points simply high-threshold loci, with one response threshold just under the maximum intensity tested and the other potentially just over this level. Indeed, behavioral thresholds at pure points were lower than those at mixed points (Table 1), 
and maximum intensities tested were two to three times higher (voltage and current, respectively) than mean behavioral thresholds. (The higher increment in current at maximum intensities reflects a voltage-dependent decrease in impedance, characteristic of brain tissue.) Further, it is unlikely that pure points were due to the presence of stronger interfering responses at these loci, which may have obscured the second behavior. The similarity in the maximum intensities tested at pure and mixed points reflects a similar degree of potentially interfering motor responses at these points, since the presence of strong motor responses was the criterion for terminating the increasing voltage series. Moreover, since attack and threat are not incompatible, it is unlikely that one behavioral response could obscure the other. Both attack and threat were seen simultaneously during stimulation of mixed points, even at high stimulation levels where responses became extremely strong.

The above results, indicating a considerable double dissociation between biting attack and threat, suggest the existence of distinct neural systems mediating these responses. This suggestion is further supported by the finding, consistent with previous reports (Roberts \&
Table 1

Number of Loci Producing Different Responses and Stimulation Parameters

\begin{tabular}{lrrrr}
\hline & & \multicolumn{2}{c}{ Mean Thresholds (V/mA) $\dagger$} & Maximum \\
\cline { 3 - 4 } \multicolumn{1}{c}{ Loci } & $\mathrm{N}$ & Attack & Threat & Intensity $\dagger \dagger$ \\
\hline $\begin{array}{l}\text { Pure } \\
\text { Response* }\end{array}$ & 58 & $(20) 5.1 / .57$ & $(38) 5.0 / .58$ & $11.0 / 1.46$ \\
$\begin{array}{l}\text { Mixed } \\
\text { Response }\end{array}$ & 23 & $6.0 / .69$ & $7.2 / .74$ & $11.8 / 1.51$ \\
$\begin{array}{l}\text { Negative } \\
\text { Overall }\end{array}$ & 25 & & & $14.1 / 2.00$ \\
\hline
\end{tabular}

*Numbers in parentheses indicate number of pure attack and pure threat points, respectively.

tVoltage and current measures were taken at the midpoint of the pulses.

††Mean maximum stimulation intensity tested (volts/milliampere.

Kiess, 1964; Wasman \& Flynn, 1962), that threat points were located more medially than those producing attack, indicating a regional differentiation between attack and threat systems (mean lateral placement for threat points $=1.3 \mathrm{~mm}$, for attack $=1.9 \mathrm{~mm} ; \mathrm{t}=4.11, \mathrm{df}=102$, $\mathrm{p}<.001$ ). Indeed, mixed response points may best be
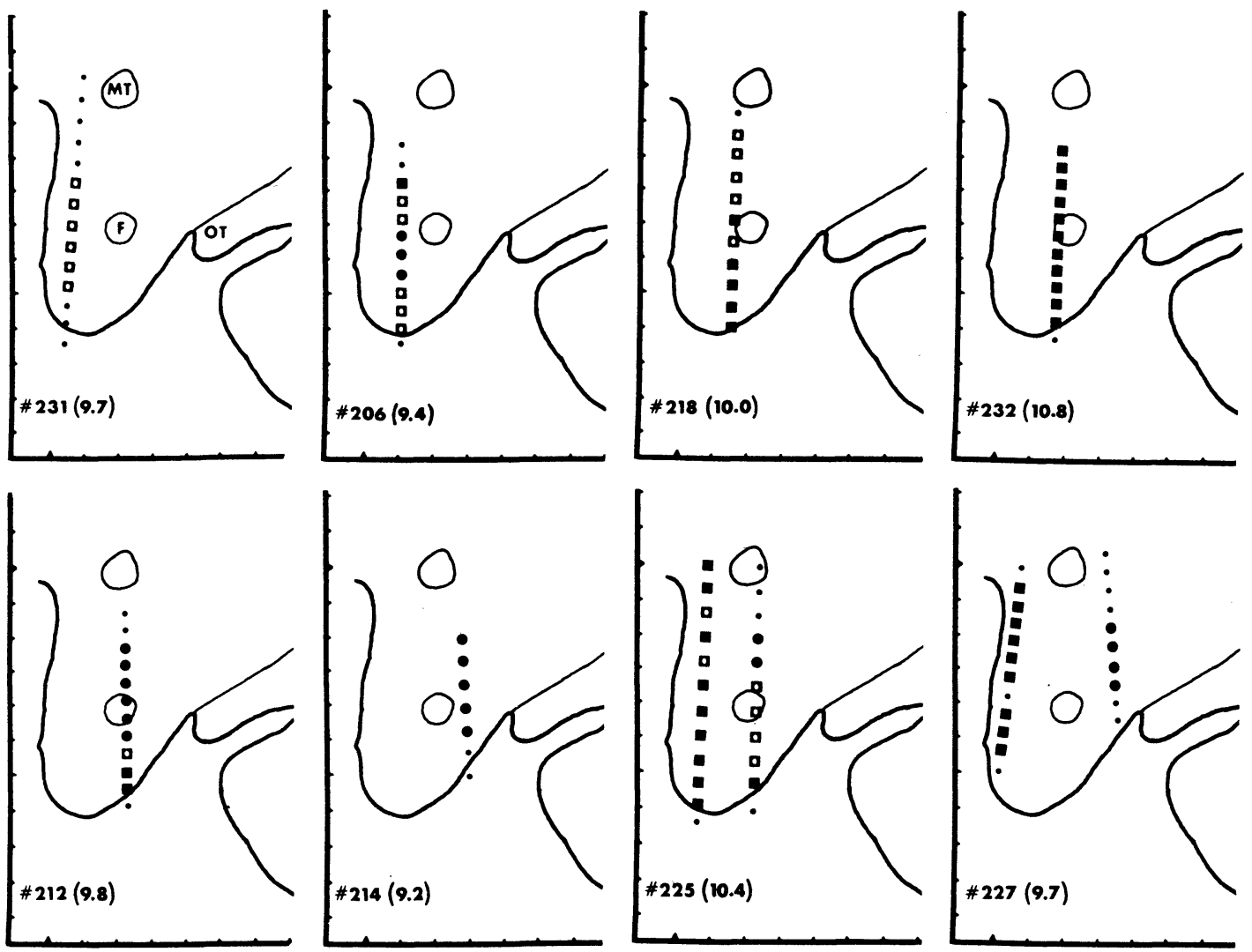

-ATTACK

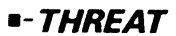

-ATTACK \& THREAT

- NEGATIVE

Figure 1. Frontal diagrams of the cat hypothalamus showing locations of stimulation points for each animal, and behaviors elicited at each point. All points are plotted on plane A10.0 of the atlas of Snider and Niemer (1961) to facilitate comparison. Numbers in parentheses indicate actual frontal placement for each cat. F, columna fornicis; MT, fasciculus mamillothalamicus; OT, tractus opticus. 


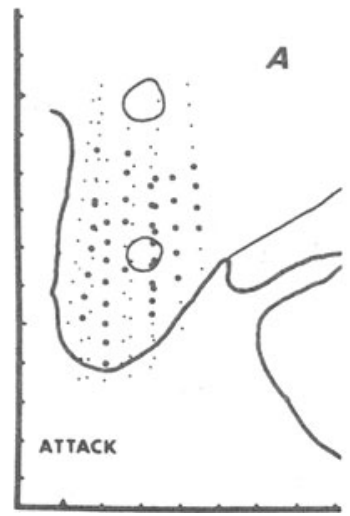

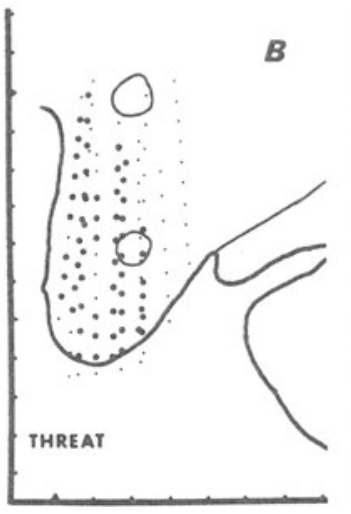

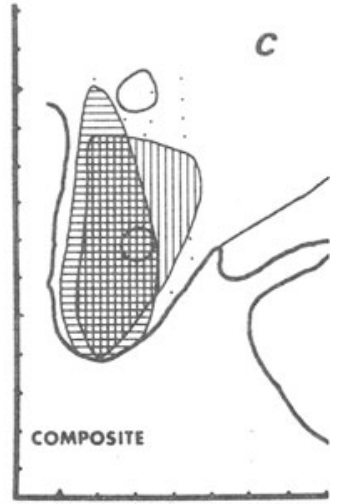

Figure 2. Composite diagrams showing all stimulation points and effective areas for different responses. Large dots indicate positive points, small dots, negative points. A. Positive and negative points for attack. B. Positive and negative points for threat. C. Effective areas for threat (horizontal lines), and attack (vertical lines). Small dots indicate negative points excluded from effective areas. viewed as resulting from current spread between response mechanisms, due to the incidental anatomical association of distinct neural systems, rather than from a homogeneous response substrate or from functional linkages between response systems. A post-hoc analysis of the conditional probabilities of attack and threat supports this view. This analysis reveals that the conditional probabilities of obtaining attack or threat, given the other response, are no greater than the overall probabilities of obtaining the respective behaviors. Thus: $\mathrm{p}($ attack/threat $)=$ Number mixed points/total number threat points $=23 / 61=.38 ; \mathrm{p}($ attack $)=$ Number attack points/total number of points $=43 / 106=.41$; $\mathrm{p}($ threat/attack $)=$ Number mixed points/total number attack points $=23 / 43=.53 ; \mathrm{p}($ threat $)=$ Number threat points/total number of points $=61 / 106=.58$. If the responses resulted from activation of a homogeneous substrate, or if functional linkages exist between response systems, the conditional probabilities of obtaining one response, given the other, should be significantly greater than the overall probabilities of the responses. Thus, while there is certainly overlap between the neural mechanisms for attack and threat, the present data provide no support for the notion of a homogeneous response mechanism mediating these behaviors. In fact, the finding that loci producing threat are located significantly more medially than those producing attack demonstrate at least a partial anatomical segregation of these response mechanisms.

\section{Analysis of Response Zones \\ Based on Pooled Anatomical Data}

Figure 2 shows composite diagrams of all stimulation points, and the stimulation-defined "effective areas" for attack and threat. Outlines of "effective areas" were drawn to include all positive points, and exclude distant negatives. Of the 94 stimulation points within these effective areas, 38 produced only threat, 20 only attack, 23 produced both responses, and 13 were completely negative. Analysis of these data reveals a negative association between attack and threat in these regions. There are fewer mixed points than would be predicted on the basis of chance associations of independent probabilities $\left(\chi^{2}\right.$ test for goodness of fit, $\chi^{2}=4.72$, $\mathrm{df}=1, \mathrm{p}<.05$ ). Examination of Figure 2 reveals that this negative association is due, at least in part, to the most medial zone from which only threat was obtained, and the most lateral, from which only attack could be elicited. These results are consistent with the finding that threat points lie more medial than attack points and indicate that in extreme medial and lateral areas there is no, or virtually no, overlap between the two behaviors. Even within the central overlap zone shown in Figure 2C, one finds no evidence for a specific association between attack and threat. Of the 57 points in this zone, 23 produced both attack and threat, 21 yielded only threat, 9 produced pure attack, and 4 produced neither response. These values do not differ significantly from those predicted on the basis of chance associations of independent probabilities (24.7, 19.3, 7.3 , \& 5.7, respectively). The present analysis not only argues against the notion of a homogeneous hypothalamic mechanism mediating attack and threat behaviors in the cat, but demonstrates a negative association between responses, due at least in part to anatomical segregation of response mechanisms.

\section{Between-Animal Comparisons}

Composite diagrams based on pooled data, such as those shown in Figure 2, can provide important information on the relative locations of behavioral systems. The results of the present study, however, indicate that such maps must be interpreted with caution. Examination of the vertical extent of individual "effective zones" for attack and threat, based on the distributions of positive loci shown in Figure 1, reveals that there is considerable interanimal variability in the vertical location and extent of these zones, even between nearby electrodes. To the extent that animal-to-animal variability exists in the precise location of behavioral systems, composite maps based on pooled data give spuriously large estimates of the size of effective substrates within a given animal (Roberts, 1969). Thus, the composite map (Figure 2C) overestimates the 
vertical extent of individual zones by $46 \%$ for biting attack (range $0 \%$ to $166 \%$ for the seven electrode tracks extending vertically throughout the composite attack area), and $55 \%$ for threat (range $0 \%$ to $267 \%$ for the seven electrodes extending throughout the threat zone). While some of the animal-to-animal variability observed in the present study may have been due to slight differences in frontal placements in different animals, individual electrodes deviated by a mean of less than $.4 \mathrm{~mm}$ from the median frontal placement, which is less than twice the thickness of the stimulation electrodes, and about 1/15th of the frontal extent of the cat hypothalamus. In any event, one could never be assured that different electrodes are in exactly the same plane; a composite map always includes a range of frontal placements.

Some additional variability in the vertical size and extent of individual effective zones may be due to slight differences in mediolateral placement. Regardless of the source of variability, the fact remains that, for a given electrode in given mediolateral and frontal planes, the composite map overestimates by up to $267 \%$ the actual experimental values obtained.

As a consequence of its overestimate of the size of effective substrates, a composite diagram would also overestimate the degree of anatomical overlap between different behaviors for a given animal. Thus, for the seven individual electrode tracks extending vertically throughout the composite overlap zone, the composite map predicts a $100 \%$ overlap between the anatomical distributions of attack and threat loci in the vertical dimension. Examination of individual "effective zones" for attack and threat, for these seven animals, reveals that the mean anatomical overlap between the distributions of points producing these two responses was only $44 \%$ (range $0 \%$ to $100 \%$ ). Thus, the composite map overestimated the mean percentage overlap, for individual animals, by more than two times.

These results are reminiscent of reports on stimulation of the motor cortex, which indicate considerable intersubject variability in the anatomical representation of selected muscle groups (Asanuma \& Sakata, 1967; Penfield \& Jasper, 1954; Woolsey et al., 1951). Indeed, examination of individual stimulation maps from the study of Asanuma and Sakata (1967) indicates that a composite map, based on a number of animals, can overestimate the size of the relevant area for a given animal by more than ten times, and at the same time, substantially overestimate the degree of overlap between muscle groups.

\section{SUMMARY AND CONCLUSIONS}

We found that hypothalamic areas from which electrical stimulation could elicit biting attack and threat, while partially overlapping, could be anatomically differentiated. Threat alone was elicited by stimulation of the most medial sites, while activation of the most lateral produced only attack. Even within the middle area of overlapping representation, many loci produced only one of the two responses. Further, the proportions of points in the overlap area producing one response, both responses, or no response, were not significantly different from those predicted on the basis of incidental anatomical associations of independent systems. These results strongly support the suggestions of Roberts (1969) and Wise (1968) that the hypothalamus contains anatomically and functionally discrete, but partially interdigitating, systems for specific behaviors.

We further showed that a composite stimulation map, based on anatomical data pooled across animals, substantially overestimated the size of individual substrates, and the degree of anatomical overlap between response systems. In view of this potential overestimate of the size and overlap of behavioral systems based on pooled data, and of the demonstrated functional differentiation between behaviors, one must view with caution arguments for functional nonspecificity in neurobehavioral systems, based on the overlap of neural substrates.

The present study indicates that different behaviors could be reliably elicited from different stimulation loci in a given animal, within the same testing environment, and both within and between stimulation sessions. These results suggest that biting attack and threat elicited by hypothalamic stimulation result from the activation of stable behavioral systems, rather than from the elicitation of a general motivational state, with the specific behavior obtained depending on previous experience, or on the dispositional state of the animal.

\section{REFERENCES}

Andersson, B. The effect of injections of hypertonic $\mathrm{NaCl}$ solutions into different parts of the hypothalamus of goats. Acta Physiologica Scandinavica, 1953, 28, 188-201.

Asanuma, H., \& Sakata, H. Functional organization of a cortical efferent system examined with focal depth stimulation in cats. Journal of Neurophysiology, 1967, 30, 35-54.

Bergquist, E. H. Output pathways of hypothalamic mechanisms for sexual, aggressive and other motivated behaviors in the opposum. Journal of Comparative and Physiological Psychology, 1970, 70, 389-398.

Berntson, G. G. Blockade and release of hypothalamically and naturally elicited aggressive behaviors in cats following midbrain lesions. Journal of Comparative and Physiological Psycholoy, 1972, 81, 541-554

Berntson, G. G., \& Hughes, H. C. Medullary mechanisms for eating and grooming behaviors in the cat. Experimental Neurology, 1974, 44, 255-265.

Chi, C. C., \& Flynn, J. P. Neuroanatomic projections related to biting attack elicited from hypothalamus in cats. Brain Research, 1971, 35, 49-66.

Doty, R. W. Electrical stimulation of the brain in behavioral context. Annual Review of Psychology, 1969, 20, 289-320.

Fisher, A. E. Chemical and electrical stimulation of the brain in the male rat. In $R$. S. Gorski and R. E. Whalen (Eds.), The brain and gonadal function. Berkeley \& Los Angeles: University of California Press, 1966.

Flynn, J. P., Edwards, S. B., \& Bandler, R. J. Changes in sensory and motor systems during centrally elicited attack. Behavioral Science, 1971, 16, 1-19.

Grossman, S. P. A neuropharmacological analysis of hypothalamic and extrahypothalamic mechanisms concerned with the regulation of food and water intake. Annals of the New York Academy of Science, 1969, 157, 902-912. 
Hess, W. K. The functional organization of the diencephalon New York: Grune and Stratton, 1957.

Leibowitz, S. F. Hypothalamic alpha- and beta-adrenergic systems regulate both thirst and hunger in the rat. Proceedings of the National Academy of Science, 1971, 68, 332-334.

Mogenson, G. J., \& Huang, Y. H. The neurobiology of motivated behavior. Progress in Neurobiology, 1973, 1,55-83.

Penfield, W., \& Jasper, H. Epilepsy and the functional anatomy of the human brain. Boston: Little, Brown, 1954.

Roberts, W. W. Are hypothalamic motivational mechanisms functionally and anatomically specific? Brain, Behavior, and Evolution, 1969, 2, 317-342.

Roberts, W. W. Hypothalamic mechanisms for motivational and species-ty pical behavior. In R. E. Whalen, R. F. Thompson, M. Verzeano, and N. M. Weinberger (Eds.), The neural control of behavior. New York: Academic Press, 1970. Pp. 175-206.

Roberts, w. W., Berquist, E. H., \& Robinson, T. C. L. Thermoregulatory grooming and sleep-like relaxation induced by local warming of preoptic area and anterior hypothalamus in opossum. Journal of Comparative and Physiological Psychology, 1969, 67, 182-188.

Roberts, W. W., \& Kiess, H. O. Motivational properties of hy pothalamic aggression in cats. Journal of Comparative and Physiological Psychology, 1964, 58, 187-193.

Roberts, W. W., Steinberg, M. L., \& Means, L. W. Hypothalamic mechanisms for sexual, aggressive, and other motivational behaviors in the opossum, Didelphis virginiana. Journal of Comparative and Physiological Psychology, 1967, 64, 1-15.
Snider, R. J., \& Niemer, W. T. A stereotaxic atlas of the cat brain. Chicago: University of Chicago Press, 1961.

Valenstein, E. S., Cox, V. C., \& Kakolewski, J. W. Reexamination of the role of the hypothalamus in motivation. Psychological Review, 1970, 77, 16-31.

Waldbillig, R. J. Attack, eating, drinking, and gnawing elicited by electrical stimulation of rat mesencephalon and pons. Journal of Comparative and Physiological Psychology, 1975, in press. Wasman, M., \& Flynn, J. P. Directed attack elicited from hy pothalamus. Archives of Neurology, 1962, 6, 220-227.

Wise, R. A. Hypothalamic motivational systems: Fixed or plastic neural circuits. Science, 1968, 162, 377-379.

Woodworth, C. H. Attack elicited in rats by electrical stimulation of the lateral hypothalamus. Physiology and Behavior, 1971, 6, 345-353.

Woolsey, C. N., Settlage, P. H., Meyer, D. R., Spencer, W. Pinto-Hamuy, T., \& Travis, A. M. Patterns of localization in precentral and "supplementary" motor areas and their relation to the concept of a premotor area. Research Publications of the Association for Nervous and Mental Diseases, 1951, 30, 238-264.

(Received for publication October 17,1974; revision received November 25,1974 .) 